Research Paper

\title{
Fault Detection of Gear Using Spectrum and Cepstrum Analysis
}

KIRAN VERNEKAR, HEMANTHA KUMAR* and K V GANGADHARAN

Department of Mechanical Engineering, National Institute of Technology Karanataka, Surathkal, Mangalore, India

(Received on 18 December 2014; Revised on 08 June 2015; Accepted on 18 June 2015)

\begin{abstract}
This paper presents an experimental investigation on damage detection of internal combustion (IC) engine gear box using conventional vibration spectrum and cepstrum analysis. Experiment was carried out on two stroke internal combustion engine gearbox without considering the combustion. Vibration signals were collected for healthy as well as defective gear condition. The signals were analysed in time domain, frequency domain and cepstrum plots for fault detection. An experimental result demonstrates the dynamic behaviour in frequency domain, which is dominated by gear mesh frequency (GMF) and its harmonics.Based on the experimental results obtained, spectrum and cepstrum analysis can be effectively used for fault prediction of machine components.
\end{abstract}

Key words: Damage Detection; Gear Box; Spectrum Analysis; Cepstrum Analysis; Internal Combustion Engine

\section{Introduction}

Condition monitoring of rotating machine elements based on vibration signal processing has an attractive research area. Vibration analysis is most important technique which is extensively used for condition monitoring of machinery systems. Vibration measuring instruments generate complex time series waveform, which contains specific signatures. If these vibration signatures are properly analysed, it is very easy to predict the unexpected failure of machinery system. Spectrum and cepstrum analysis are machine diagnostic methods used for monitoring any rotary machine equipment. These methods can demonstrate certain information on operating condition that determines the malfunctions such as rotary imbalance, shaft misalignment, bearing and gear damages. Generally a perfect gear in mesh produces a perfectly smooth sinusoidal signal in time domain and a prominent gear running frequency, gear mesh frequencies and its harmonics in frequency domain. Faults in gear system such as broken tooth, misalignment will result in change in pattern of frequency spectrum, which indicates the development of fault or failure of components.

Few researchers carried out study on machine diagnostics. Randall (1973) demonstrated simplified interpretation of gear diagnostics using cepstrum. El Morsy and Achtenová (2014) experimentally presented a study on diagnostics of an artificial pitting defect in a vehicle gearbox using cepstrum analysis. Vernekar et al. (2014) used fast Fourier transforms (FFT) and wavelet transforms (WT) for fault detection of the gearbox. For machine diagnostics application spectrum and cepstrum analysis are generally applied and well suited (Satyam et al., 2013; Nacib et al., 1963; Amit and Khalid, 2012). Vernekar et al. (2014) carried out an experimental investigation of fault diagnosis of bearing used in IC engine using machine learning approach. Gear box has a complex structure and early fault detection and its location can prevent unexpected failure of machinery system, which in turn improves its safety. Significant work has been carried out in the area of fault detection using various signal processing techniques, but investigations on an engine

*Author for Correspondence: E-mail: hemanta76@gmail.com; Tel: +918242473681 
gear box with vibration signal are very limited. This paper attempts to demonstrate a method for fault detection of the gearbox using spectrum and cepstrum analysis. The experimental results demonstrate that the proposed method can well diagnose the fault in the gear box.

\section{Spectrum and Cepstrum Analysis}

Time domain represents the dynamic responses dependence on the interaction of many components of the system.It helps to analyse the amplitude and phase information of the vibration time signal to detect the fault of any rotating machinery system. In other words, it is particularly used to analyse signals from bearing and gear defects with unsteady and short transient impulses (McFadden, 1987). Diagnosis of time series response is a difficult task; relating linking a change to a particular component requires the manual calculation of the repetition frequency based on the time difference observed between feature points. The spectrum and cepstrum analysis on the other hand have potential to reveal the diagnostic information from time domain signals.

\section{Spectrum Analysis}

Fourier transform (FT) based spectral analysis is the dominant analytical tool for frequency domain analysis. Fourier transform is most widely used in vibration signal analysis. It simply converts given signal from time domain to frequency domain by integrating the given function over the entire time period. FT for the angular frequency $\omega=2 \pi f$ and time $t$ is given by,

$$
X(\omega)=\int_{-\infty}^{+\infty} x(t) e^{-j \omega t} d t \text { foward transform }
$$

Inverse transform(2)

Where $X(\omega)$ is the Fourier transform of the signal $x(t)$. Using these equations, a signal $x(t)$ can be transformed into the frequency domain and back again. The FT is well suited to analyse stationary periodic functions which will exactly repeat themselves once every period, without modification.Fourier transform is particularly used to convert a function from the continuous time to the continuous frequency domain, where as Fast Fourier Transform (FFT) is an efficient algorithm to compute the discrete Fourier transform and it's inverse with considerable savings in computational time. FT technique earned much of the importance in processing stationary signal. FFT is the one of the its extension of it. Frequency domain analysis not only detects the fault but also indicates the cause of the defect in the machinery system (Vernekar et al., 2014).

\section{Cepstrum Analysis}

A cepstrum is considered as forward Fourier transformation of the logarithm of a spectrum. It is therefore defined as the spectrum of a spectrum. The name 'Cepstrum' is derived by reversing first four letters of word 'spectrum'. Cepstrum analysis is also known as quefrency or cepstral analysis. The independent variable on the $\mathrm{x}$-axis of spectrum is frequency. The independent variable of a cepstrum is called "quefrency". The word quefrency is derived from the word frequency by interchanging its first three letters' with its second three letters. The cepstrum $x(t)=\overline{\text { was }} 2$ the logarithmic power spectrum, thus the calculation of cepstrum involves the inverse Fourier transform of the natural logarithm of a spectrum (Randall, 1982). Given a real signal $\mathrm{x}(\mathrm{n})$, different cepstrum forms can be expressed as follows,

The real Cepstrum of a signal $x(n)$ :

$$
c(n)=\frac{1}{2 \pi} \int_{-\pi}^{\pi} \log X\left|\left(e^{j \omega}\right)\right| e^{j \omega n} d \omega
$$

The complex Cepstrum of a signal $x(n)$ :

$$
c(n)=\frac{1}{2 \pi} \int_{-\pi}^{\pi} \log X[(\omega)] e^{j \omega n} d \omega
$$

The power Cepstrum of a signal $x(n)$ :

$$
c(t)^{2}=\frac{1}{2 \pi}\left|\int_{-\pi}^{\pi} \log \right| X(\omega)\left|e^{j \omega n} d \omega\right|^{2}
$$

In this the present investigation real cepstrum 
has been used to reveal the periodicity in frequency domain. Fig. 1 depicts the relationship between spectrum and cepstrum. Table 1 gives the comparison of terms used in spectrum and cepstrum analysis.

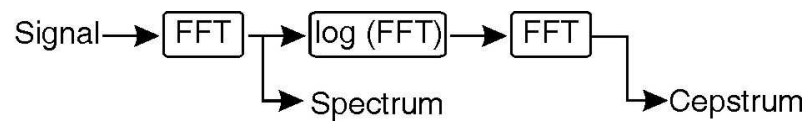

Fig. 1: The relationship between a spectrum and a cepstrum

Table 1: Comparisons of terms used in spectrum and cepstrum analysis

\begin{tabular}{ll}
\hline Spectrum analysis & Cepstrum analysis \\
\hline Spectrum & Cepstrum \\
Frequency $(\mathrm{Hz})$ & Quefrency (second) \\
Harmonic & Rahmonic \\
Filter & Lifter \\
\hline
\end{tabular}

\section{Experimental Studies}

An engine test rig was developed to study the fault detection of gear used in IC engine. The details of the experimental setup and procedure will be discussed in the following subsections.

\section{Experimental Setup Description}

In this research, Kawasaki Bajaj (KB-100) two stroke spark ignition engine gearbox was considered for the study. Figs. 2 and 3 illustrate the line diagram and experimental test setup used for the fault detection of gear box used in IC engine. The experiments were conducted on the KB-100 engine without considering the combustion. Generally, the engine conditions are monitored at idle speed, which is about $1000 \mathrm{rpm}$. Hence in the current experiment engine speed was maintained at $1100 \mathrm{rpm}$ in 4 th gear position. Here the engine speed refers to the rotational speed of the crank. A torque level of $30 \mathrm{Nm}$ at output shaft was maintained and digital Tachometer was used to monitor crankshaft speed. This condition was maintained for all test cases. The engine was driven by 3 HP DC motor. The data has been acquired using NI-9234 data acquisition (DAQ) card and analysed using LabVIEW software from National Instruments
(NI). Piezoelectric accelerometer with an operating frequency range between 1 to $5000 \mathrm{~Hz}$ was used to pick-up vibration signals (magnitude of acceleration with respect to time). The sampling frequency of 5 $\mathrm{KHz}$ was used to collect the data for 1 second.

\section{Experimental Procedure}

In this experiment, the vibration signal was acquired from a healthy gear condition at constant crank shaft speed of $1100 \mathrm{rpm}(18 \mathrm{~Hz})$, which is considered as the baseline. In second test, artificial fault is inducedby removing one tooth from the gear and corresponding vibration readings were extracted. The Fig. 4 shows the gear conditions and Fig. 5 shows the sectional view of the KB-100 gear box used for research purposein the present study.

In the case of gearbox vibrations, gear mesh frequency (GMF) is important, which is generated due to modulation phenomena. In faulty condition of a gear, magnitude of GMF increases which is the indication of the defective condition of gear (Lokesh et al., 2011). The characteristic vibration frequencies of the engine are listed in Table 2.

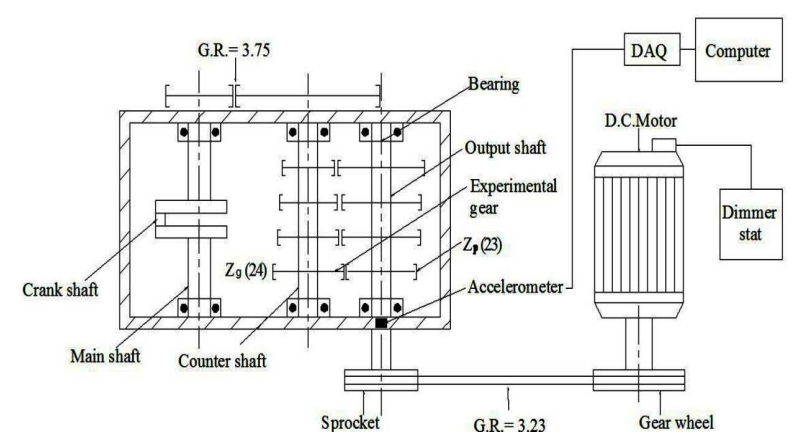

Fig. 2: Line diagram of experimental setup

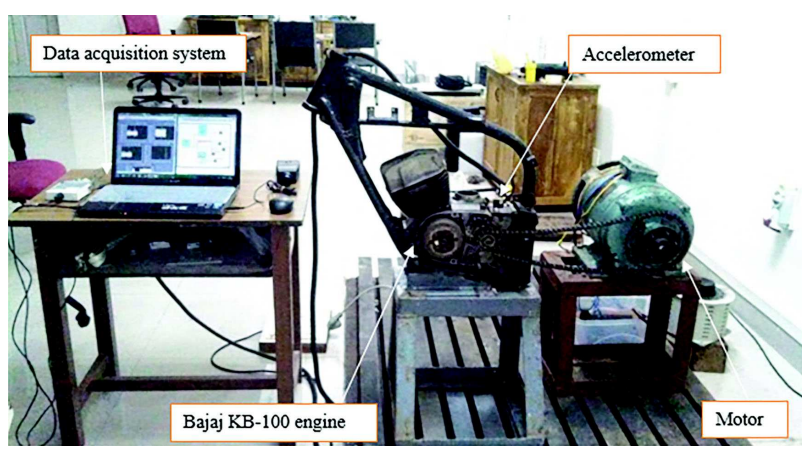

Fig. 3: Experimental set-up of gear box fault detection 
Table 2: Characteristic vibration frequency at engine running speed

\begin{tabular}{lc}
\hline Parameters & Frequency $(\mathrm{Hz})$ \\
\hline Gear rotational frequency & 4.87 \\
Pinion rotational frequency & 5 \\
Gear mesh frequency (GMF) & 117 \\
Crank shaft rotating frequency $\left(\mathrm{f}_{\mathrm{s}}\right)$ & 18 \\
\hline
\end{tabular}

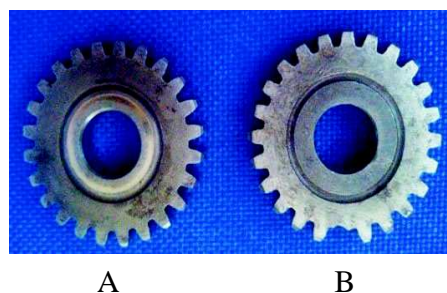

Fig. 4: Gear conditions (A) Healthy gear (B) Gears with simulated artificial fault

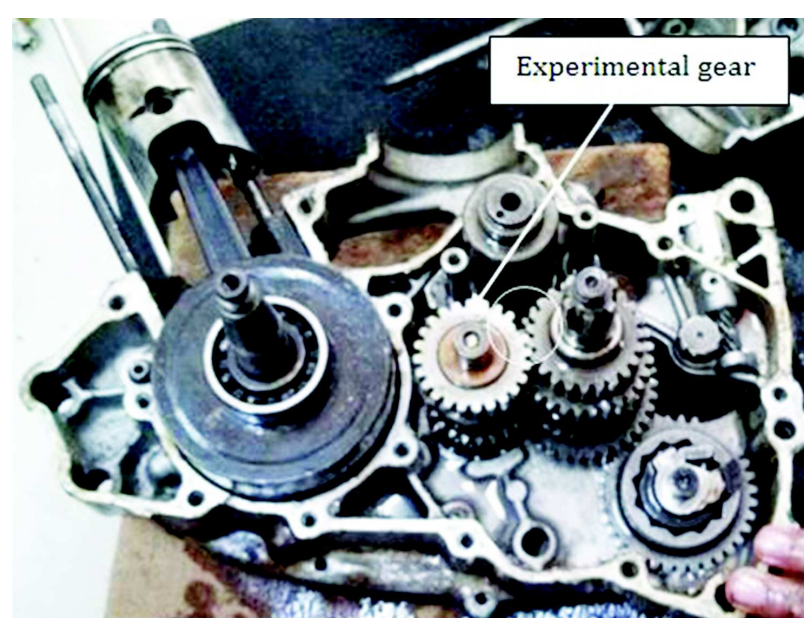

Fig. 5: Sectioned view of engine

\section{Results and Discussion}

\section{Experiment 1: Healthy Gear}

In this section, experimental result on fault detection of gearbox using conventional vibration technique, based on the FFT and Cepstrum is demonstrated. The experimental results with healthy condition of gear are shown in Fig. 6. Fig. 6A represents the dynamic response in time domain and Fig. 6B illustrates frequency spectrum pertaining to healthy gear condition. In the spectrum plot the peak frequency component at $18 \mathrm{~Hz}$, which is the running frequency of the crank shaft rotation (fs), is observable. Another peak is observed at $36 \mathrm{~Hz}$ (2xfs), which is due to secondary force of reciprocating mass and most of the rest of the peaks are multiples of crank shaft rotating frequencies. Gear mesh frequency at $117 \mathrm{~Hz}$ can also be observed in the same frequency spectrum plot. It can be noticed from frequency spectrum that the vibration of the crank shaft has highest influence on the spectrum of the gearbox, which is dominant among all the frequencies while the gear rotating frequency $(4.87 \mathrm{~Hz})$ has not been noticed in the frequency spectrum. The cepstrum of the healthy gear as shown in Fig. 6C, has an obvious peak at $0.055 \mathrm{~s}$ corresponding to harmonics of $18 \mathrm{~Hz}$.

\section{Experiment 2: Gear with simulated artificial fault}

Fig. 7 demonstrates vibration response of faulty gear. From time domain response as shown in Fig. 7A, it can be observed that the acceleration level is increased with reference to Fig. 6A. The frequency spectrum of the vibration signal of the gear with a fault is shown in Fig. 7B. It has a spectral peak at $18 \mathrm{~Hz}$ along with several harmonics. The increase in the amplitude of GMF $(117 \mathrm{~Hz})$ can be visualized in frequency spectrum. The magnitude of GMF is increased from 0.025 for health gear to $0.11 \mathrm{~m} / \mathrm{s}^{2}$ for the gear with fault, which indicates a fault in the gearbox. Fig. 7C depicts cepstrum plot of the gear with a simulated fault, which has obvious peak at $0.055 \mathrm{~s}$ corresponding to harmonics of $18 \mathrm{~Hz}$. Another Peak peak at 0.0085 $\mathrm{s}$ corresponding to harmonics of Gear mesh frequency $(117 \mathrm{~Hz})$ can also be noticed, which also indicates a fault. Rahmonics corresponding to $18 \mathrm{~Hz}$ and $117 \mathrm{~Hz}$ are observed from the cepstrum plot.

\section{Conclusion}

This paper presents investigation on fault detection of gear box of an IC engine using two vibrations signal processing techniques: Spectrum and Cepstrum analyses. The presence of fault in rotating machinery usually generates strong harmonics and sidebands. A time domain technique for vibration signal analysis gives overall vibration level but do not provide any diagnostic information. Spectrum analysis is a promising technique to analyse fundamental 


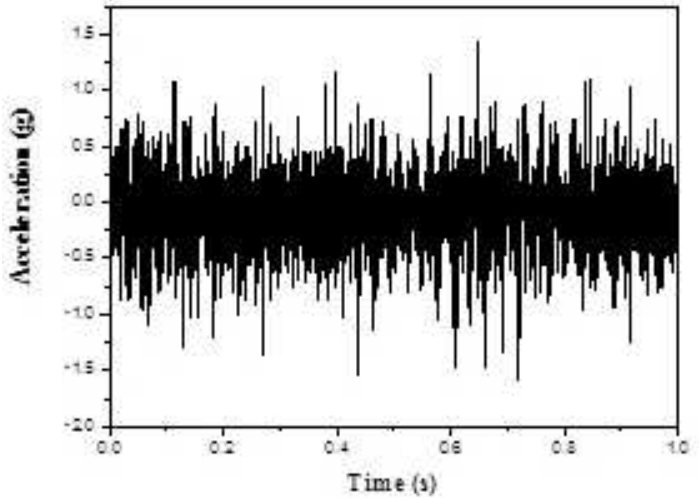

A

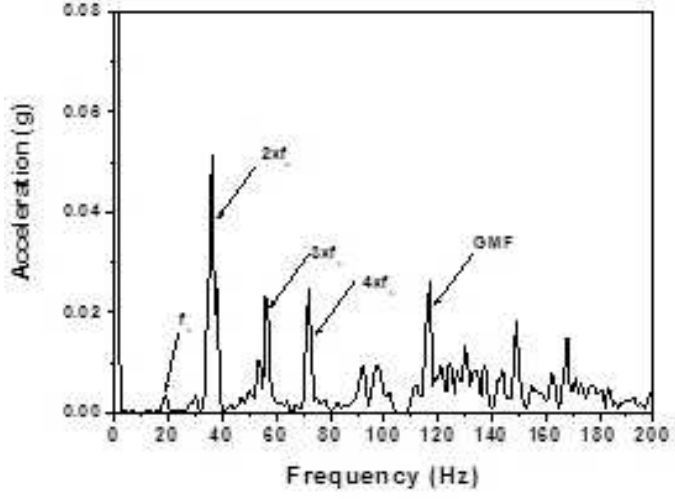

B

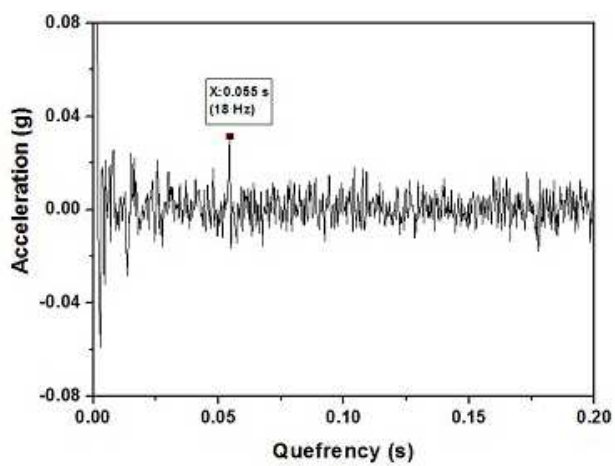

C

Fig. 6: Vibration response of a healthy gear. (A) Vibration response in Time domain (B) Frequency spectrum (C) Cepstrum

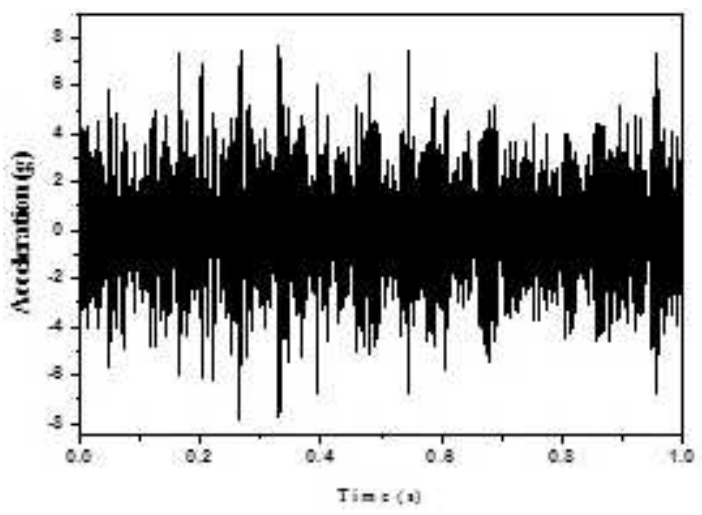

A

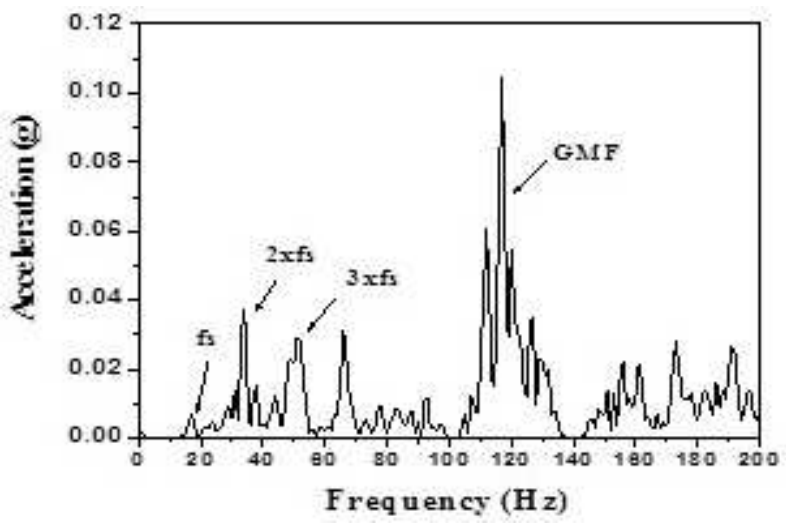

B

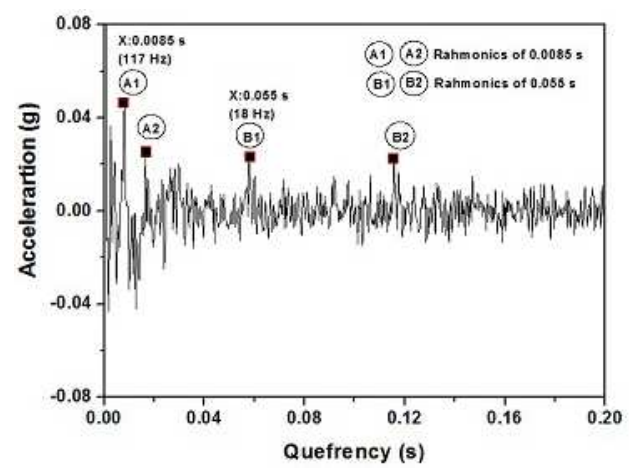

C

Fig. 7: Vibration response of a faulty gear. (A) Vibration response in Time domain (B) Frequency spectrum (C) Cepstrum 
frequency content and Cepstrum analysis is an effective tool which can be used for the detection of periodicity in a spectrum. Both the spectrum and cepstrum analysis are suitable techniques to identify and distinguish the fault frequencies.

\section{References}

Aherwar A and Khalid S (2012) Vibration analysis techniques for gearbox diagnostic: a review International Journal of Advanced Engineering Technology 3 4-12

El Morsy M and Achtenová G (2014) Vehicle Gearbox Fault Diagnosis Based On Cepstrum Analysis International Journal of Mechanical, Aerospace, Industrial and Mechatronics Engineering 8 1547-1553

Vernekar K, Kumar H and Gangadharan K V (2014) Gear Fault detection using vibration analysis and continuous wavelet transform Procedia Materials Science 5 1846-1852

Vernekar K, Kumar H and Gangadharan K V (2014) Fault diagnosis of deep groove ball bearing through discrete wavelet features using support vector machine International Journal of COMADEM 17 31-37

Lokesha M, Majumder M C, Ramachandran K P, Fathi Khalid and Raheem Abdul (2011) Fault diagnosis in gear using

\section{Acknowledgments}

The authors acknowledge the funding support from SOLVE: The Virtual Lab @ NITK (www.solve.nitk. ac.in) and experimental facility provided by Centre for System Design (CSD): A Centre of excellence at NITK-Surathkal.

wavelet envelope power spectrum International Journal of Engineering, Science and Technology 3 156-167

Nacib L, Komi M P and Sakhara S (1963) Detecting gear tooth cracks using cepstral analysis in gearbox of helicopters International Journal of Advanced Engineering Technology 5 139-145

McFadden P D (1987) A revised model for the extraction of periodic waveforms by time domain averaging Mechanical systems and signal processing 183-95

Randall R B (1973) Cepstrum Analysis and Gearbox Fault Diagnosis. Brüel and Kjaer instruments Inc. Copenhagen.

Randall R B (1982) Cepstrum analysis and gearbox fault-diagnosis Maintenance Management International 3 183-208

Satyam M, Rao V S and Devy C G (2013) Cepstrum Analysis: An Advanced Technique in Vibration Analysis of Defects in Rotating Machinery Defence Science Journal 44 53-60. 